\title{
Study on Unbalanced Power Flow in Distribution Network with Distributed Generators of Power Grid
}

\author{
Zhong-Zheng Tong ${ }^{1}$, Yang-Zi Sun ${ }^{2}$, Wen-Bin Liu ${ }^{1}$, Chu-Ran Deng ${ }^{1}$ \\ ${ }^{1}$ Information Center of Guangdong Power Grid Co., Ltd., Guangzhou, Guangdong 510080, China \\ ${ }^{2}$ Shanwei Power Supply Bureau of Guangdong Power Grid Co., Ltd., Shanwei, Guangdong 516600, China
}

How to cite this paper: Tong, Z.Z., Sun, Y.Z., Liu, W.B., Deng, C.R. (2018) Study on Unbalanced Power Flow in Distribution Network with Distributed Generators of Power Grid. Journal of Applied Mathematics and Computation, 2(8), 285-290.

http://dx.doi.org/10.26855/ja mc.2018.08.001 *Corresponding author: Zhong-Zheng Tong, Information Center of Guangdong Power Grid Co., Ltd., Guangzhou, Guangdong 510080, China Email: zhongzheng818@163.com

\section{Abstract}

With rapid increase in wind power penetration into the power grid, unbalanced power flow in distribution network with distributed generators is becoming increasingly important to power sysytem. Due to the variability and uncertainty of wind, such traditional unbalanced power flow analysis method for distributed power supply limit the use of the existing tools for decision-making under uncertain conditions. As a result, unbalanced power flow analysis method, which provides information on uncertainty associated with wind power analysis, is gaining increased attention. This paper presents a novel hybrid intelligent algorithm for deterministic unbalanced power flow in distribution network with distributed generators that utilizes a combination of Cauchy particle swarm optimization (CPSO) and fuzzy ARTMAP (FA) network, which is optimized by using PSD-BPA. The performance of the proposed model is assessed utilizing wind power data from the Baolihua wind farm in Southern China. Unbalanced power flow in distribution network with distributed generators is researched. The typical static load model is established and the impact of distributed generators on feeder voltage profile is analyzed under unbalanced loads. The simulation results are given. References for the global planning of unbalance compensation in distribution network can be provided by the study.

\section{Keywords}

Power integration, power quality, Cauchy particle swarm optimization, unbalanced power flow.

\section{Introduction.}

In modern power system, a large impact asymmetric load can lead to unbalanced problem, which threat to safety and stable for power distribution network. In modern power system, a large impact asymmetric load can lead to unbalanced problem, which threat to safety and stable for power distribution network. As of June 2014, the total installed capacity of distributed generator (DG) are exceeded 200MW in a southern coastal city in China, and it is expected that the cumulative installed capacity of wind power can reached 300MW and 380MW accounting for $38 \%$ and $45 \%$ of the proportion of the load in this area in 2015 and 2016.New energy is more and more widespread concern in all aspects and the proportion of new energy in the power grid is more and more rapidly[1]. The large power grid International Commission defines a distributed power supply, which is about the size of a $5 \mathrm{MW} 100 \mathrm{MW}$. Usually, connected to a power network, is a non - planned or a centralized power production mode. When the distributed power supply develops to a certain scale, while providing clean energy and also has a negative impact on the power grid. Especially, with the wind energy 
and other new energy into the grid scale from small to large, and large capacity wind power plant directly into the transmission network, which has a great impact on the regional power grid. Previously, the most common impact is harmonic pollution, voltage fluctuations, etc.. Due to the distributed power into the regional power grid, development to the present, that had an impact to the stability of the system and the reactive power regulation [2-4].

\section{Put Forward The Question}

Wind speed change, the status of the power generation equipment, as well as the inherent characteristics of the distributed generator will lead to power output fluctuations, resulting in voltage fluctuations, voltage flicker, voltage mutations beyond the standard value. Distributed generator connected will bring a series impact on the power grid, the most important impact is the flow direction of the trend. In system, the trend is no longer one direction flow, and the traditional power flow algorithm is not satisfied because no consideration the grid-connected of various forms distributed power supply[5-7].

\section{The Power Network Modelling}

In the three-phase power trend algorithm, use the model to reflect the characteristics of the three-phase structure and the node admittance matrix reflect the coupling relationship between phase and phase. Taking the A phase node as an example, the self-admittance of the A phase node is the sum of the admittance of the A phase line connected to the same bus bar. The mutual-admittance is the negative number of the sum admittance, which the A phase connected with this bus bar and the other connected.

Three phase unbalanced power trend of distribution network with distributed generator can be expressed by $\Delta S=J \Delta U$ and $\Delta S=[\Delta P, \Delta Q]^{T}$, the local power grid in each distributed power unit is equivalent to the $\mathrm{PQ}$ node. According to the given wind speed and power factor, the active power and reactive power of the wind turbine generator are calculated.

The power imbalance matrix of a system with $n+1$ node is expressed in the following, where there are $l$ nodes of the $\mathrm{PQ}, n-l$ nodes of the PV, and one balanced nodes.

The power unbalance value of the PQ node can be expressed as the difference between the power given value of the node and the actual power calculated by the current voltage, and can be expressed as:

$$
\left\{\begin{array}{l}
\Delta P_{i}^{\nabla}=P_{i}^{\nabla}-\left|U_{i}^{\nabla}\right| \sum_{j=1}^{n} \sum_{l}\left|U_{j}^{l}\right|\left(G_{i j}^{\nabla l} \cos \theta_{i j}^{\nabla l}+B_{i j}^{\nabla l} \sin \theta_{i j}^{\nabla l}\right) \\
\Delta Q_{i}^{\nabla}=Q_{i}^{\nabla}-\left|U_{i}^{\nabla}\right| \sum_{j=1}^{n} \sum_{l}\left|U_{j}^{l}\right|\left(G_{i j}^{\nabla l} \sin \theta_{i j}^{\nabla l}-B_{i j}^{\nabla l} \cos \theta_{i j}^{\nabla l}\right)
\end{array}\right.
$$

where, $i=1,2, \ldots, n-1, \quad p=a, b, c, \quad l=a, b, c$

For the PV node, the node voltage amplitude is given, not as a variable. At the same time, the point cannot be given reactive power in advance, so the reactive power imbalance of this point will be lost the constraint effect. Therefore, there is no need to calculate the reactive power equation related to the PV node in the iteration process[9].

$$
\Delta P_{i}^{\nabla}=P_{i}^{\nabla}-\left|U_{i}^{\nabla}\right| \sum_{j=1}^{n} \sum_{l}\left|U_{j}^{l}\right|\left(G_{i j}^{\nabla l} \cos \theta_{i j}^{\nabla l}+B_{i j}^{\nabla l} \sin \theta_{i j}^{\nabla l}\right)
$$

where, $i=1,2, \ldots, n-1, \quad p=a, b, c, \quad l=a, b, c$

Considering the reactive power of the wind power plant is affected by the node voltage, in order to further improve the model, use the $\Delta U$ values obtained to correct the voltage values of each node, and the next iteration is carried out until the convergence condition is satisfied.

\section{Typical Static Load Model}

\section{A. The Model Design}

The traditional power grid are radiation, after accessing the distributed generator, the transmission power is reduced, due to the support of the output power of the distributed power supply, the voltage of each load node is improved. And the number of voltage is raised are relate to the position and the total capacity of the distributed generator [13].

Define the original load on the power grid is positive load, the distributed generator is negative load, and the line length is $L, \quad P_{D G i}$ and $Q_{D G i}(i=1,2, \ldots, 1 n(2 \mathrm{n}))$ are respectively expressed active power and reactive power of distributed generator as shown in Fig. 1: 


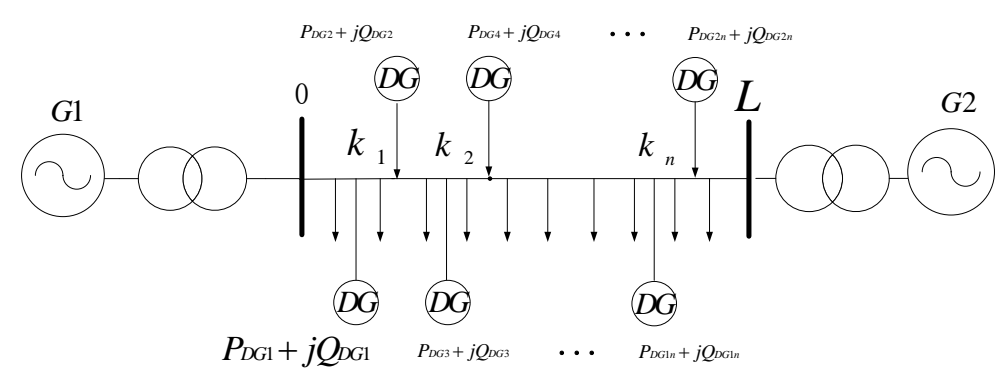

Fig.1 Feeder with distributed generators

\section{B. The Model Building}

Taking the load increasing distribution model as an example, initial load is $P_{0}+j Q_{0}$, among them $P_{0}$ and $Q_{0}$ are the active power and reactive power of the load. Where $\varpi \theta$ express any point of the line. Where $\eta$ is the slope of the load distribution curve $\eta=\eta_{1}+j \eta_{2}$, among them $\eta_{1}$ and $\eta_{2}$ are curve slope of the active load and the reactive load, $\eta_{1}, \eta_{2}>0$. The load at any point of the $\varpi \theta$ is:

$$
\left\{\begin{array}{l}
P(\varpi \theta)=P_{0}-\eta_{1} \varpi \theta \\
Q(\varpi \theta)=Q_{0}-\eta_{2} \varpi \theta
\end{array}\right.
$$

The increasing load distribution is shown in Fig. 2:

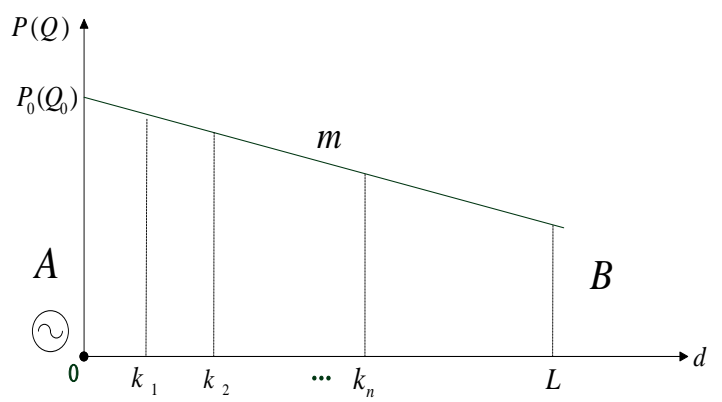

Fig. 2 Feeder of decreasingly distributed load with DGs

The $n$ distributed generator arranged $k_{1}, k_{2} \ldots k_{n} \in[0, L]$ on the line. Let $k_{0}=0, k_{n+1}=L$, when the any note of the feeder $\varpi \theta \in\left[k_{i-1}, k_{i}\right](i=0,1,2 \ldots, n+1)$, the front and back load of the $\varpi \theta$ notes are respectively as:

$$
\begin{gathered}
\left\{\begin{array}{l}
P_{0-\varpi \theta}=P_{0} \varpi \theta+\frac{1}{2} \eta_{1} \varpi \theta^{2}-\sum_{j=1}^{i-1} P_{D G j} \\
Q_{0-\varpi \theta}=Q_{0} \varpi \theta+\frac{1}{2} \eta_{2} \varpi \theta^{2}-\sum_{j=1}^{i-1} Q_{D G j}
\end{array}\right. \\
\left\{\begin{array}{l}
P_{\varpi \theta-L}=P_{0}(L-\varpi \theta)+\frac{\eta_{1}}{2}\left(L^{2}-\varpi \theta^{2}\right)-\sum_{j=i}^{n} P_{D G j} \\
Q_{\varpi \theta-L}=Q_{0}(L-\varpi \theta)+\frac{\eta_{2}}{2}\left(L^{2}-\varpi \theta^{2}\right)-\sum_{j=i}^{n} Q_{D G j}
\end{array}\right.
\end{gathered}
$$

\section{The Effect Of Distributed Generator}

At present, the most of China's power grid is still in the radial chain structure. This paper presents a novel hybrid intelligent algorithm for deterministic unbalanced power flow in distribution network with distributed generators that utilizes a combination of Cauchy particle swarm optimization(CPSO) and fuzzy ARTMAP (FA) network, which is optimized by using PSD-BPA. As an example, taking the power trend diagram of a certain area the three-phase unbalanced trend distribution of the distributed generator are analyzed, as shown in Fig. 3: 


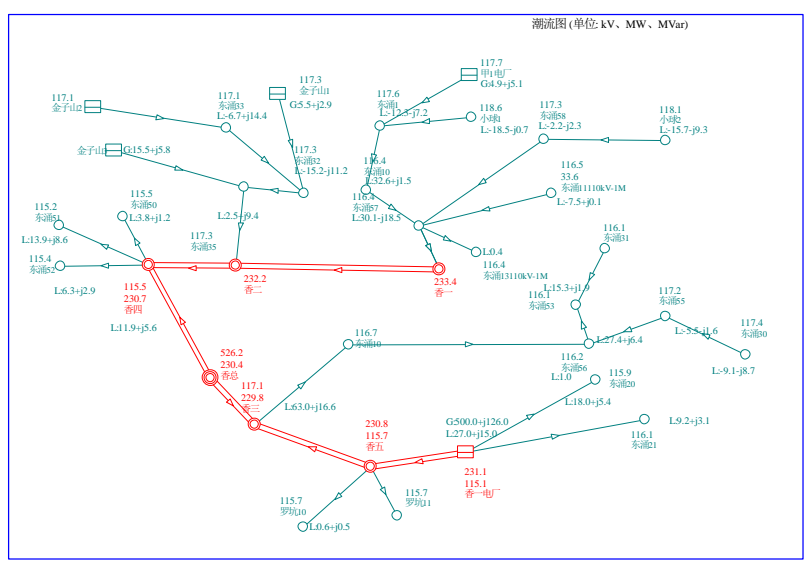

Fig. 3 Diagram of a region power flow

In the diagram, the red line represented the voltage is $220 \mathrm{kV}$. Related parameters are defined as: rated voltage is $u_{N}=110 \mathrm{kV}$, line length is $L=10 \mathrm{~km}$, unit length resistance is $r=0.20 \Omega / \mathrm{km}$, reactance is $x=0.45 \Omega / \mathrm{km}$, active load at the beginning of the line is $P_{0}=0.2 \mathrm{MW}$, reactive load is $Q_{0}=0.1 \mathrm{Mvar}$, line starting voltage is $u_{0}=110.5 \mathrm{kV}$, the slope of the load curve is $\eta_{1}=\eta_{2}=0.28, t_{1}=t_{2}=-0.25$, all the power factor of the grid connected in distributed generator is $\cos \theta=0.95$.

The distributed generator capacity certain and the access point is close to the starting position is less impact for the voltage. Near the end position, the impact on the voltage is greater. When the distributed generator is incorporated into the end of the line, the lowest point of the voltage is no longer the end of the line, the voltage stability analysis needs to reconsider the position of the lowest voltage. The distributed generator capacity is effect to feeder each phase voltage, the capacity is shown in Table 1:

Table 1. Capacities of DGs

\begin{tabular}{ccc}
\hline Number & The location of DG & $\begin{array}{c}\text { The capacity of } \\
\text { DG }(\cos \theta=0.95)\end{array}$ \\
\hline 1 & DG & $P_{D G 1}=10 \mathrm{MW}, P_{D G 2}=10 \mathrm{MW}$ \\
2 & $k_{1}=3 \mathrm{~km}, k_{2}=8 \mathrm{~km}$ & \\
3 & $k_{1}=3 \mathrm{~km}, k_{2}=8 \mathrm{~km}$ & $P_{D G 1}=20 \mathrm{MW}, P_{D G 2}=20 \mathrm{MW}$ \\
4 & $k_{1}=3 \mathrm{~km}, k_{2}=8 \mathrm{~km}$ & $P_{D G 1}=30 \mathrm{MW}, P_{D G 2}=30 \mathrm{MW}$ \\
\hline
\end{tabular}

The simulation results are shown in figure 4:

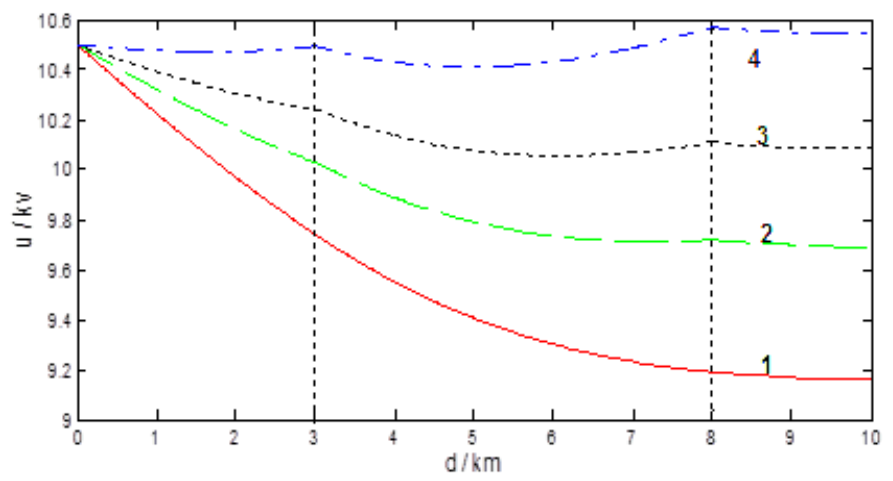

Fig.4 Voltage profile of feeder under different capacities of DGs 
The quantitative analysis to the curve, and solve the voltage offset $V_{\text {offset }}$ at the key points of the curve in Figure 4:

For curve 1: When it is no DGs, the voltage offset $V_{\text {offset }}=0$

For curve 2:

$$
\begin{aligned}
& V_{\text {offset }}\left(k_{1}\right)=\lambda \frac{\left|u_{\sigma \theta}-u_{N}\right|}{u_{N}} \times 100 \%=2 \times \frac{|10.51-10.3|}{10.0} \times 100 \%=3.2 \% \\
& V_{\text {offset }}\left(k_{2}\right)=\lambda \frac{\left|u_{\pi \theta}-u_{N}\right|}{u_{N}} \times 100 \%=1 \times \frac{|9.72-10.0|}{10.0} \times 100 \%=2.81 \% \\
& V_{\text {offset }}(L)=\lambda \frac{\left|u_{\sigma \theta}-u_{N}\right|}{u_{N}} \times 100 \%=1 \times \frac{|9.69-10.0|}{10.0} \times 100 \%=3.06 \%
\end{aligned}
$$

For curve 3:

$$
\begin{aligned}
& V_{\text {offset }}\left(k_{1}\right)=\lambda \frac{\left|u_{\pi \theta}-u_{N}\right|}{u_{N}} \times 100 \%=2 \times \frac{|10.84-10.3|}{10.0} \times 100 \%=10.8 \% \\
& V_{\text {offset }}\left(k_{2}\right)=\lambda \frac{\left|u_{\pi \theta}-u_{N}\right|}{u_{N}} \times 100 \%=2 \times \frac{|10.51-10.3|}{10.0} \times 100 \%=4.2 \% \\
& V_{\text {offset }}(L)=\lambda \frac{\left|u_{\sigma \theta}-u_{N}\right|}{u_{N}} \times 100 \%=1 \times \frac{|10.29-10.0|}{10.0} \times 100 \%=2.9 \%
\end{aligned}
$$

For curve 4:

$$
\begin{aligned}
& V_{\text {offset }}\left(k_{1}\right)=\lambda \frac{\left|u_{\pi \theta}-u_{N}\right|}{u_{N}} \times 100 \%=2 \times \frac{|10.99-10.3|}{10.0} \times 100 \%=13.8 \% \\
& V_{\text {offset }}\left(k_{2}\right)=\lambda \frac{\left|u_{\omega \theta}-u_{N}\right|}{u_{N}} \times 100 \%=2 \times \frac{|10.87-10.3|}{10.0} \times 100 \%=11.4 \% \\
& V_{\text {offset }}(L)=\lambda \frac{\left|u_{\sigma \theta}-u_{N}\right|}{u_{N}} \times 100 \%=2 \times \frac{|10.84-10.3|}{10.0} \times 100 \%=10.08 \%
\end{aligned}
$$

As can be seen, when the distributed generator capacity is 10MW (in Fig. 4 and Curve 2), the voltage offset is small, and the voltage distribution is relatively stable. With the increase of the distributed generator capacity, the voltage is gradually raised, even more serious over voltage (in Fig. 4 and Curve 4).

Based on the above analysis, we can know that the distributed generator will produce a certain impact on the distribution of the power grid, which determined by the location and relative capacity of the distributed generator. When three-phase load unbalanced, the voltage distribution difference of each phase is increased, so it is necessary reasonable allocation to distributed generator in order to effectively control the voltage distribution.

\section{Conclusion}

This paper discussed that the situation of power grid unbalanced load flow distribution with the distributed generator, the feeder voltage distribution of radial power network is studied, a typical static load model is established, and the expression of voltage distribution is derived based on this model. Taking the power grid in a certain area as an example, by simulation, the effect of the position and relative capacity of the distributed generator on the voltage distribution of each phase is analyzed. It provides a basis that the rational planning of the distributed generator and use it to adjust power grid three-phase unbalance load.

\section{References}

[1] Ciric R, Nouri H, Terzija V. Impact of distributed generators on arcing faults in distribution networks [J]. Generation, Transmission \& Distribution, IET, 2011, 5(5): 596-601.

[2] Richard E B. Impact of Smart Grid on distribution system design [C]. IEEE Power and Energy Society General Meeting, Pittsburgh, July 2008:1-4.

[3] Rahimi F, Ipakchi A. Demand response as a market resource under the Smart Grid paradigm [J]. IEEE trans. Smart Grid, June 2010, 1(1): 82-85.

[4] Li F X, Qiao H B, Sun H, et al. Smart transmission grid: vision and framework [J]. IEEE trans. Smart Grid, 2010, 1(2): $168-171$. 
[5] El-Khattam W, Sidhu T S. Restoration of directional overcurrent relay coordination in distributed generation sys tems utilizing fault current limiter [J]. IEEE trans. Power Delivery, April 2008, 23(2): 576-585.

[6] Hazel T G, Hiscock N, Hiscock J. Voltage regulation at sites with distributed generation [J]. IEEE trans. Industry Applications, 2008, 44(2):445-454.

[7] Viawan F A, Karlsson D. Coordinated voltage and reactive power control in the presence of distributed generation[C]. Power and Energy Society General Meeting, July 2008: 1-6.

[8] Li Zh X, Sun Q Y, Zhang H G. Voltage profile of feeder with multiple distributed generators[C]. CIAC'2009, Nanjing, China, 2009.

[9] Kamh M Z, Iravani R. A unified three-phase power-flow analysis model for electronically coupled distributed energy resources [J]. IEEE trans. Power Delivery, 2011, 2(26): 899-909.

\section{Author's Introduction}

TONG Zhong-zheng (MS'2011), was born in P.R.China, on Feb. 26, 1980, is a senior electric engineer. He received M.S. degree in China Northeastern University. His research interests include the power system dispatch, electronic system simulation, and the monitoring, analysis and assessment of modern power quality.

Tel: +86 13500081267

Email: zhongzheng818@163.com

SUN Yang-zi (MS'2011), was born in P.R.China, on Mar. 15, 1984, is an electric engineer. She received M.S. degree in China Liaoning Shihua University. Her research interests include the power system-level design, electronic system simulation, and the monitoring, analysis and assessment of modern power quality.

Tel: +86 13500082487

Email: csf7734806@163.com

LIU Wen-bin(MS'1993), was born in P.R.China, on July 19, 1967, is with the China Southern Grid Information Center of Guangdong Power Grid Co., Ltd., Guangzhou, China.

Tel: +8613609779182

Fax: 020-83125962

Email: liuwenbin@xxzx.gd.csg.cn

DENG Chu-ran (MS'2016), was born in P.R.China, on Feb. 18, 1993, is an data engineer. She received M.S. degree in Hong Kong University of Science and Technology. Her research interests include the electric power informatization, big data technology, data science, data analysis.

Tel: +86 15521133797

Email: dengchuran@foxmail.com 